\title{
AN AFFINE CONTROL METHOD FOR OPTIMAL DYNAMIC ASSET ALLOCATION WITH TRANSACTION COSTS*
}

\author{
GIUSEPPE CARLO CALAFIORE ${ }^{\dagger}$
}

\begin{abstract}
In this paper, we present a novel and computationally efficient approach to constrained discrete-time dynamic asset allocation over multiple periods. This technique is able to control portfolio expectation and variance at both final and intermediate stages of the decision horizon and may account for proportional transaction costs and intertemporal dependence of the return process. A key feature of the proposed method is the use of a linearly parameterized class of feedback control policies, which permits us to obtain explicit analytic expressions for the portfolio statistics over time. These expressions are proved to be convex in the decision parameters, and hence, under these control laws, the multistage problem is formulated and solved by means of efficient tools for quadratic or second-order-cone convex programming.
\end{abstract}

Key words. dynamic optimization, strategic asset allocation, multistage decision problems, risk control, convex optimization, portfolio optimization, transaction costs

AMS subject classifications. 91B28, 49M37, 90C25, 93E25

DOI. $10.1137 / 080723776$

1. Introduction. Institutional investors manage their strategic mix of asset classes over time to achieve favorable returns in spite of uncertainties. A fundamental issue in this context is to maintain risk under control while achieving the desired return targets. The well-known Markowitz mean-variance portfolio model [23, 24] provides a useful tool to compute such a tradeoff between return and risk, but it assumes a single time period for the determination of the optimal allocation. In realistic situations, the asset mix needs instead to be rebalanced many times over the investment horizon, and hence the decision maker actually faces a difficult constrained dynamic optimization problem that should take into account conditional decisions based on future market behavior. Therefore, for an investor with a long-term investment horizon, the question arises whether the optimal multiperiod portfolio has any relationship to optimal holdings in a single-period setting. In his seminal works [25, 26], Merton proposes a continuous-time stochastic dynamic programming approach to the multiperiod allocation problem. Despite the fact that this model is quite complicated and unsuitable for efficient practical implementation, Merton manages to show that if the objective is maximization of a utility function and if the assets' expected returns and covariances are constant over the decision horizon, then the asset allocations are also constant and equal to the one-period solutions for a mean-variance investor; that is, there is no intertemporal hedging demand in this case.

However, Merton's formulation considers only maximization of the terminal wealth (i.e., it ignores the path and risk taken to arrive at the terminal wealth) and does not take into account cost of transactions or the possibility of imposing constraints on the portfolio composition at the final and intermediate stages. When these realistic features are added to the model, the myopic one-period approach is no longer equivalent to the optimal multiperiod one, and hence an investment advantage may be gained

\footnotetext{
*Received by the editors May 9, 2008; accepted for publication (in revised form) May 8, 2009; published electronically June 25, 2009.

http://www.siam.org/journals/sicon/48-4/72377.html

$\dagger$ Dipartimento di Automatica e Informatica, Politecnico di Torino, Torino 10129, Italy (giuseppe. calafiore@polito.it).
} 
by employing a decision model that fully exploits the intertemporal structure of the problem. Moreover, multiperiod problems in the presence of transaction costs and constraints are in general not tractable analytically by means of the optimal control techniques employed, for instance, in $[7,8,10,25,26]$.

The mainstream computational model for dealing with multiperiod decision problems in the presence of uncertainty is currently provided by multistage stochastic programming; see, e.g., [3, 4, 5, 14, 31, 33] and the many references therein. However, while stochastic programming provides a conceptually sound framework for posing multistage decision problems, from the computational side it results in being impervious to an exact and efficient numerical solution [32]. The key difficulty in the stochastic programming formulation comes from the fact that the stage decisions are actually conditional decision rules or "policies" that define which action should be taken in response to past outcomes. To model the conditional nature of the problem in some "tractable" way, a discretization of the decision space is typically introduced by constructing a "scenario tree," and this scenario tree may grow exponentially if an accurate and representative discretization is needed; see, e.g., [12]. On the other hand, if branching is kept low in the scenario tree, the resulting discretization cannot be guaranteed to be a reliable representation of reality. These computational difficulties are witnessed by the fact that most multiperiod problems discussed in the literature deal with few securities over only two or three periods.

In the specific context of financial allocation, a classical stochastic programming method based on Benders' decomposition is proposed in [11], and techniques for construction of scenario trees are discussed, for instance, in [27, 30, 35]. Scenario-based stochastic programming models for portfolio optimization have also been recently proposed in $[17,28]$. A survey with theoretical analysis of multiperiod models based on scenario trees is provided in [34].

In this paper, we propose a different route to multistage allocation, which prescinds from the use of decision trees or sample paths and which leads to explicit convex programming models that can be solved globally and efficiently. We achieve these goals by considering a restricted class of decision policies that are affine functions of the past return innovations. Within this setting, we obtain exact and explicit expressions for portfolio expectations and volatilities at all stages of the decision horizon, and these expressions result in being affine or convex quadratic functions of the decision parameters. This approach thus leads to computationally tractable optimization problems (convex quadratic or second-order-cone programming problems) whose solutions provide suboptimal policies, since affinely parameterized recourse functions constitute only a subclass of the set of possible recourse relations. This suboptimality is, however, largely counterweighted by numerical tractability of the ensuing optimization programs, which enables application of multistage techniques to real-world investment endeavors. The idea of using affine decision rules has been inspired by a similar "linearly adjustable variables" technique that has seemingly been first studied in [2] in the context of robust optimization. Adjustable variables have also been proposed in the context of model predictive control in [19]. More generally, affine decision policies are reminiscent of the classical linear feedback laws used for control of dynamical systems, and indeed control techniques are currently attracting much interest for their applications to financial problems; see, for instance, [1, 15, 29].

Affine recourse for dynamic financial allocation problems has been introduced by this author in [9]. This work extends the results of [9] in several ways. First, here we remove the hypothesis of serially independent returns and develop the decision model in full generality with respect to the stochastic market model (the independence 
hypothesis is reintroduced later in section 5 as a special case). Second, we use control rules with possibly full memory of the past return history, contrary to [9] where only one-period memory was considered. Lastly, the model discussed in the present work takes into account the possible presence of proportional transaction costs.

The paper is organized as follows. Section 2 contains definitions and problem setup. In section 3 the affinely parameterized control function class is introduced, and explicit expressions for the time evolution of portfolio expectations and covariances are derived. Section 3.2 provides upper and lower bounds for the total expected cost of transaction under the considered control policy. Explicit convex programming representations are given in section 4 . Sliding-horizon implementations of the decision model are discussed in section 4.2, whereas sampling-based evaluation of market statistics is treated in section 4.3. Section 5 considers the special but practically relevant situation of efficient markets. Under this hypothesis, a key result is given in Lemma 5.1 which provides a recursive analytic expression for the portfolio variance under the considered control policy. The results of some numerical tests are described in section 6 , while conclusions are drawn in section 7 .

Notation. If $x \in \mathbb{R}^{n, 1}$, then $\operatorname{diag}(x)$ denotes a diagonal matrix having the entries of $x$ on the diagonal. $A^{\top}$ denotes the transpose of matrix $A$. The operator $\odot$ denotes the Hadamard (entrywise) product of conformably sized matrices. $I_{n}$ and $0_{n}$ denote the identity matrix and the zero matrix in $\mathbb{R}^{n, n}$, respectively. For a random vector $x$, $\mathrm{E}\{x\}$ denotes the expected value of $x$ and $\operatorname{var}\{x\} \doteq \mathrm{E}\left\{(x-\mathrm{E}\{x\})(x-\mathrm{E}\{x\})^{\top}\right\}$ its covariance matrix. We shall denote with an over bar the expectation of random quantities and with a tilde the centered quantities, i.e., the quantities with the expectation subtracted, that is, $\bar{x} \doteq \mathrm{E}\{x\}, \tilde{x} \doteq x-\bar{x}$.

2. Setup and preliminaries. Consider an investment problem involving $n$ assets or asset classes $\left\{a_{1}, \ldots, a_{n}\right\}$, which may include cash, over a decision horizon of $T$ periods, where the $k$ th period starts at time $k-1$ and ends at time $k$. We denote with $x_{i}(k)$ the Euro value of the portion of the investor's total wealth invested in security $a_{i}$ at time $k$. The portfolio at time $k$ is the vector

$$
x(k) \doteq\left[\begin{array}{lll}
x_{1}(k) & \cdots & x_{n}(k)
\end{array}\right]^{\top}
$$

with $x(0)$ the initial portfolio. The investor's total wealth at time $k$ is

$$
w(k) \doteq \sum_{i=1}^{n} x(k)=\mathbf{1}^{\top} x(k)
$$

where 1 denotes a vector of ones. At the end of each period, the investor has the opportunity to adjust his/her investment by rebalancing the portfolio composition. Specifically, we denote with $x^{+}(k)$ the portfolio composition just after the adjustment $u(k)$ occurred at time $k$ :

$$
x^{+}(k) \doteq x(k)+u(k)
$$

where $u(k) \doteq\left[u_{1}(k) \cdots u_{n}(k)\right]^{\top}$ is the vector of portfolio adjustments. A value of $u_{i}(k)>0$ indicates that the portfolio content in asset $a_{i}$ is increased by $u_{i}(k)$ Euros (by buying this asset), whereas $u_{i}(k)<0$ indicates that the portfolio content in asset $a_{i}$ is decreased by $u_{i}(k)$ Euros (by selling this asset). If $p_{i}(k)$ denotes the market value of $a_{i}$ at time $k$, then the (simple) return of investment in security $a_{i}$ over the 
period of time $[k-1, k]$ is $r_{i}(k) \doteq\left(p_{i}(k)-p_{i}(k-1)\right) / p_{i}(k-1)=p_{i}(k) / p_{i}(k-1)-1$, and the one-period gain of the same investment is

$$
g_{i}(k) \doteq \frac{p_{i}(k)}{p_{i}(k-1)}=r_{i}(k)+1
$$

We denote with $g(k)$ the vector collecting the asset gains and with $G(k)=\operatorname{diag}(g(k))$ the corresponding diagonal matrix. The portfolio composition then evolves in time according to the recursive equations

$$
\begin{aligned}
x^{+}(k) & =x(k)+u(k), \\
x(k+1) & =G(k+1) x^{+}(k), \quad k=0,1, \ldots, T-1 .
\end{aligned}
$$

Here, we take a standard stochastic view of the market and consider the asset gains $g(k), k=1, \ldots$, to follow a possibly nonstationary discrete-time stochastic process with finite and possibly time-varying means and covariances. (2.2) thus specifies a stochastic discrete-time system which describes the time evolution of the portfolio.

2.1. Problem statement. The purpose of our decision model is to determine investment adjustment policies (in our context, a policy is a parameterized function expressing $u(k)$ in terms of the previous market gains $g(t), t=1, \ldots, k)$ so as to attain a desired target expected return at the end of the investment horizon while maintaining the associated risk and transaction costs under control. The expected value of cumulative gross return of the investment over the whole horizon is $\mathcal{P} \doteq$ $\mathrm{E}\{w(T)\} / w(0)$. The risk associated with the investment strategy here is measured by a weighted sum of wealth variances (volatilities) at all the decision stages:

$$
\mathcal{R} \doteq \sum_{k=1}^{T} v_{k} \operatorname{var}\{w(k)\},
$$

where $v_{k} \geq 0$ are given weights. For instance, $v_{k}=1 / T, k=1, \ldots, T$, sets $\mathcal{R}$ to measure the average wealth variance over the decision horizon, whereas $v_{k}=0$, $k=1, \ldots, T-1, v_{T}=1$, sets $\mathcal{R}$ to measure only end-of-horizon variance.

Let $c_{i} \geq 0$ denote the proportional transaction cost coefficient for trading in asset $a_{i}$. The cost due to all transactions at time $k$ is given by $\sum_{i=1}^{n} c_{i}\left|u_{i}(k)\right|=\|c \odot u(k)\|_{1}$, where $\|\cdot\|_{1}$ denotes the $\ell_{1}$ vector norm and $c^{\top}=\left[c_{1} \cdots c_{n}\right]$ is the vector of unit transaction costs. The total expected transaction cost over the investment horizon is hence

$$
\mathcal{C} \doteq \mathrm{E}\left\{\sum_{k=0}^{T-1}\|c \odot u(k)\|_{1}\right\} .
$$

We consider the portfolio to be self-financing, that is, $\sum_{i=1}^{n} u_{i}(k)=0$, except for transaction costs, which are covered by newly injected cash.

Usually, constraints need to be enforced on portfolios. These constraints include, for instance, portfolio composition conditions (minimum and maximum exposure in individual assets or in groups of assets) or no-shortselling constraints. More complicated diversification constraints might be modeled via linear inequalities; see, for instance, [22]. In this paper, we include generic linear constraints in the model by 
imposing that the expected value of the updated portfolios $x^{+}(k)$ lie within a given polytope $\mathcal{X}(k)$. Further discussion on portfolio constraints is postponed to section 4.1. The multiperiod asset allocation problem (MAP) we are interested in can now be formally stated as follows:

$$
\min _{u(k) \in \mathcal{U}} \mathcal{R}+\gamma \mathcal{C} \text { (mixed risk+transaction cost objective) }
$$

subject to $\mathrm{E}\left\{x^{+}(k)\right\} \in \mathcal{X}(k), k=0, \ldots, T-1$ (portfolio composition constraints),

$$
\begin{array}{cl}
\mathbf{1}^{\top} u(k)=0, k=0, \ldots, T-1 & \text { (self-financing constraints), } \\
\mathrm{E}\{w(T)\} \geq \psi \cdot w(0) & \text { (target final return constraint), }
\end{array}
$$

where $\mathcal{U}$ is a given parametric class of strictly causal functions of $g(1), \ldots, g(T)$ to be specified in the next section, $\gamma \geq 0$ is a relative objective weight parameter, and $\psi$ is the given target final return.

We notice that considering a generic set $\mathcal{U}$ of causal recourse functions in problem (2.5) would make the problem extremely hard to solve in practice. Indeed, even the computation of the objective may not be tractable for generic $\mathcal{U}$. To the best of this author's knowledge, no polynomial-time exact method is available for treating the problem in such generality and neither are approximated schemes with guaranteed confidence bounds on the optimal objective. On the other extreme, a conventional approximation providing an upper bound on the objective of (2.5) is to restrict $\mathcal{U}$ to the class of "open-loop" strategies, that is, control functions that are actually independent of the returns. This latter approach, however, might be too coarse and fails to capture the dynamic nature of the decision problem at hand, that is, the fact that only the first decision $u(0)$ need actually be determined at time $k=0$ (the so-called "here-and-now" variable), whereas the decision maker can wait and see what progressively happens to the market before actually deciding the subsequent adjustments $u(1), \ldots, u(T-1)$.

In the next section, we introduce the class of affine recourse functions that are used in our decision model. Then, in section 3.2 we derive upper and lower bounds for the expectation of the transaction cost term $\mathcal{C}$. The combination of affine recourse strategy and upper/lower bound approximation for the transaction cost term leads to a problem formulation that is efficiently computable by means of convex programming.

3. Affine recourse strategy and portfolio dynamics. In our approach, we postulate that the control action $u(k)$ is an affine, strictly causal function of the returns' innovations:

$$
\begin{aligned}
& u(0)=\bar{u}(0), \\
& u(k)=\bar{u}(k)+\sum_{\tau=1}^{k} \Theta_{\tau}(k)[g(\tau)-\bar{g}(\tau)], \quad k=1, \ldots, T-1,
\end{aligned}
$$

where $\bar{u}(k) \in \mathbb{R}^{n}, k=0, \ldots, T-1$, are the nominal portfolio adjustments, with $u(0)=\bar{u}(0)$ representing the "here-and-now" decision variable, and $\Theta_{\tau}(k) \in \mathbb{R}^{n, n}, k=$ $1, \ldots, n, \tau=1, \ldots, k$, are additional decision variables representing market reaction matrices. We rewrite (3.1) in more compact notation as

$$
\begin{aligned}
u(0) & =\bar{u}(0), \\
u(k) & =\bar{u}(k)+\boldsymbol{\Theta}(k)[\mathbf{g}(k)-\overline{\mathbf{g}}(k)], \quad k=1, \ldots, T-1,
\end{aligned}
$$


with

$$
\boldsymbol{\Theta}(k) \doteq\left[\Theta_{1}(k) \cdots \Theta_{k}(k)\right] \in \mathbb{R}^{n, k n}, \quad \mathbf{g}(k) \doteq\left[g^{\top}(1) \cdots g^{\top}(k)\right]^{\top} \in \mathbb{R}^{n k} .
$$

Remark 1 (interpretation of the recourse policy). The affine recourse policy (3.2) bears a sound practical interpretation: The initial portfolio adjustment $u(0)=\bar{u}(0)$ is the "here-and-now" variable, describing the first "move" to be done by the investor. The successive adjustments $u(k), k=1, \ldots, T-1$, are "wait-and-see" variables whose values are assumed to be decomposed into the sum of a nominal decision $\bar{u}(k)$ and a recourse term. The nominal adjustment $\bar{u}(k)$ represents the move we would make at time $k$ if the market during periods preceding $k$ performed as expected. Since the market will never perform exactly as expected, we correct the nominal decision $\bar{u}(k)$ with a term proportional to all past market deviations from expectation. The coefficients of the correction are collected in the market reaction matrices $\Theta_{\tau}(k)$. In particular, element $\left[\Theta_{\tau}(k)\right]_{i j}$ in row $i$ and column $j$ of matrix $\Theta_{\tau}(k)$ represents the sensitivity of the control action in the $i$ th security $u_{i}(k)$ with respect to deviations from expectation of the return of the $j$ th security in the $\tau$ th period. We remark that this model with "full" past memory is a generalization of a simplified model with only one reaction matrix (i.e., $\tau=k$ only) previously developed in [9]. In the current setting, the "memory depth" of the model can actually be modulated as desired by the user by fixing to zero some of the initial blocks in the $\boldsymbol{\Theta}(k)$ matrices. Specifically, a "zero-depth" (no recourse or open-loop) model is obtained by considering $\boldsymbol{\Theta}(k)=0$, $k=1, \ldots, T-1$; a one-period depth model analogous to the one in $[9]$ is obtained by setting $\Theta_{i}(k)=0$ for $i=1, \ldots, k-1, k=2, \ldots, T-1$, whereas a generic $d$-periods depth model is obtained by setting $\Theta_{i}(k)=0$ for $i=1, \ldots, k-d, k=d+1, \ldots, T-1$.

3.1. Dynamics of portfolio statistics. Applying the control policy (3.2) to the portfolio recursion (2.1) and (2.2), we obtain the following (stochastic) recursions for the controlled portfolio:

$$
\begin{aligned}
x^{+}(k) & =x(k)+\bar{u}(k)+\mathbf{\Theta}(k)[\mathbf{g}(k)-\overline{\mathbf{g}}(k)], \\
x(k+1) & =G(k+1) x^{+}(k), \quad k=0,1, \ldots, T-1,
\end{aligned}
$$

with $\Theta(0)=0$. Our objective in this section is to obtain analytic expressions for the portfolio expectations and covariances. To this end, from repeated application of (3.4) we obtain the expression for the portfolio composition at a generic instant $k$ :

$$
x(k)=\Phi(1, k) x(0)+\Phi(k) \nu(k)+\sum_{i=1}^{k-1} \sum_{\tau=1}^{i} \Phi(i+1, k) \Theta_{\tau}(i) \tilde{g}(\tau),
$$

where

$$
\nu(k) \doteq\left[\begin{array}{c}
\bar{u}(0) \\
\bar{u}(1) \\
\vdots \\
\bar{u}(k-1)
\end{array}\right], \quad \Phi(k) \doteq[\Phi(1, k) \quad \cdots \quad \Phi(k, k)],
$$

with $\Phi(i, k), i \leq k$, a diagonal matrix of compound gains from beginning of period $i$ to end of period $k$ :

$\Phi(i, k) \doteq G(k) G(k-1) \cdots G(i)=\operatorname{diag}(\varphi(i, k)), \quad \varphi(i, k) \doteq g(k) \odot g(k-1) \odot \cdots \odot g(i)$. 
Denoting with $\left[\Theta_{\tau}(i)\right]_{c}$ the $c$ th column of matrix $\Theta_{\tau}(i),(3.5)$ is written as

$$
x(k)=\Phi(1, k) x(0)+\Phi(k) \nu(k)+\sum_{i=1}^{k-1} \sum_{\tau=1}^{i} \sum_{c=1}^{n} \Phi(i+1, k) \tilde{g}_{c}(\tau)\left[\Theta_{\tau}(i)\right]_{c} .
$$

Now let $\theta(k) \in \mathbb{R}^{n^{2} k(k-1) / 2}$ represent the vector containing all columns $\left[\Theta_{\tau}(i)\right]_{c}$ ordered lexicographically according to the triple $(i, \tau, c)$ for $i=1, \ldots, k-1, \tau=1, \ldots, i$, and $c=1, \ldots, n$, and let $\boldsymbol{\Psi}(k) \in \mathbb{R}^{n, n k(k-1) / 2}$ be the corresponding matrix formed by horizontal juxtaposition of the diagonal $\Phi(i+1, k) \tilde{g}_{c}(\tau)$ blocks in the same lexicographic order. With this notation, $x(k)$ can be written in the form

$$
x(k)=\Phi(1, k) x(0)+\boldsymbol{\Phi}(k) \nu(k)+\boldsymbol{\Psi}(k) \theta(k),
$$

where $\Phi(1, k), \boldsymbol{\Phi}(k)$, and $\boldsymbol{\Psi}(k)$ contain all terms depending on stochastic gains while decision variables are contained in $\nu(k)$ and $\theta(k)$. This formalism is useful for expressing the portfolio expectation and wealth variance, since we have

$$
\bar{x}(k)=\mathrm{E}\{x(k)\}=\bar{\Phi}(1, k) x(0)+\overline{\mathbf{\Phi}}(k) \nu(k)+\overline{\mathbf{\Psi}}(k) \theta(k)
$$

and

$$
\begin{aligned}
\operatorname{var}\{w(k)\} & =\mathrm{E}\left\{\mathbf{1}^{\top} \tilde{x}(k) \tilde{x}^{\top}(k) \mathbf{1}\right\}=\mathrm{E}\left\{\tilde{x}^{\top}(k) \mathbf{1 1}^{\top} \tilde{x}(k)\right\} \\
& =\left[\begin{array}{c}
x(0) \\
\nu(k) \\
\theta(k)
\end{array}\right]^{\top} \mathrm{E}\left\{\left[\begin{array}{c}
\tilde{\Phi}(1, k) \\
\tilde{\mathbf{\Phi}}^{\top}(k) \\
\tilde{\mathbf{\Psi}}^{\top}(k)
\end{array}\right] \mathbf{1 1}^{\top}\left[\begin{array}{c}
\tilde{\Phi}(1, k) \\
\tilde{\mathbf{\Phi}}^{\top}(k) \\
\tilde{\mathbf{\Psi}}^{\top}(k)
\end{array}\right]^{\top}\right\}\left[\begin{array}{c}
x(0) \\
\nu(k) \\
\theta(k)
\end{array}\right] \\
& =\left[\begin{array}{c}
x(0) \\
\nu(k) \\
\theta(k)
\end{array}\right]^{\top} \mathrm{E}\left\{\left[\begin{array}{c}
\tilde{\varphi}(1, k) \\
\tilde{\phi}(k) \\
\tilde{\psi}(k)
\end{array}\right]\left[\begin{array}{c}
\tilde{\varphi}(1, k) \\
\tilde{\phi}(k) \\
\tilde{\psi}(k)
\end{array}\right]^{\top}\right\}\left[\begin{array}{c}
x(0) \\
\nu(k) \\
\theta(k)
\end{array}\right] \\
& =\left[\begin{array}{c}
x(0) \\
\nu(k) \\
\theta(k)
\end{array}\right]^{\top} Q(k)\left[\begin{array}{c}
x(0) \\
\nu(k) \\
\theta(k)
\end{array}\right],
\end{aligned}
$$

where we defined

$$
\tilde{\phi}(k) \doteq \tilde{\boldsymbol{\Phi}}^{\top}(k) \mathbf{1}, \quad \tilde{\psi}(k) \doteq \tilde{\boldsymbol{\Phi}}^{\top}(k) \mathbf{1}, \quad Q(k) \doteq \mathrm{E}\left\{\left[\begin{array}{c}
\tilde{\varphi}(1, k) \\
\tilde{\phi}(k) \\
\tilde{\psi}(k)
\end{array}\right]\left[\begin{array}{c}
\tilde{\varphi}(1, k) \\
\tilde{\phi}(k) \\
\tilde{\psi}(k)
\end{array}\right]^{\top}\right\} .
$$

Notice that $Q(k)$ is a positive semidefinite matrix, since it represents a covariance. The previous algebraic derivations actually prove the following statement.

Lemma 3.1. Consider the portfolio dynamic equations under affine policy (3.3) and (3.4), and let $G(t)=\operatorname{diag}(g(t)), t=1,2, \ldots$, be a generic, possibly dependent, stochastic return process. Then, the portfolio expectation $\mathrm{E}\{x(k)\}$ is an affine function of the policy parameters $\bar{u}(t), t=0,1, \ldots, k-1$, and the total wealth variance $\operatorname{var}\{w(k)\}$ is a convex quadratic function of these parameters and of $\boldsymbol{\Theta}(t)$, $t=1, \ldots, k-1$.

Notice that although (3.7) and (3.8) show that the expected portfolio is affine in the decision variables and that the wealth variance is convex and quadratic in 
the variables, these functions are not easy to obtain exactly in practice, since they depend on parameters $\bar{\Phi}(1, k), \overline{\boldsymbol{\Phi}}(k), \overline{\mathbf{\Psi}}(k)$, and $Q(k)$ that are difficult to evaluate for generic and serially correlated return processes. We shall discuss in section 4.3 an approximated technique for computing the parameters $\bar{\Phi}(1, k), \overline{\boldsymbol{\Phi}}(k), \overline{\mathbf{\Psi}}(k)$, and $Q(k)$ in full generality. Also, a key result given in section 5 shows how to compute portfolio expectations and covariances exactly under a standard efficient market hypothesis.

3.2. Bounds on expected transaction cost. The result in the previous lemma implies that, under affine policy, the risk term $\mathcal{R}$ in (2.3) can be explicitly expressed as a convex quadratic function of the model decision variables $\bar{u}(k)$ and $\boldsymbol{\Theta}(k)$, and that the final expected wealth $\mathrm{E}\{w(T)\}$ is explicitly given as an affine function of the $\bar{u}(k)$ 's. In order to obtain an explicit convex approximation to problem (2.5), however, we still need to elaborate on the expected transaction cost term $\mathcal{C}$ in $(2.4)$, which cannot be expressed in analytic form. In this section, we develop computable upper and lower bounds for the expected transaction cost. First notice that, using the recourse rule (3.2) in (2.4), we have that

$$
\mathcal{C}=\sum_{k=0}^{T-1} \mathrm{E}\left\{\|c \odot(\bar{u}(k)+\boldsymbol{\Theta}(k) \tilde{\mathbf{g}}(k))\|_{1}\right\} .
$$

The following lemma holds.

LEMMA 3.2 (upper and lower bounds on $\mathcal{C}$ ). The expected transaction cost term in (3.9) is upper and lower bounded as $\underline{\mathcal{C}} \leq \mathcal{C} \leq \overline{\mathcal{C}}$, where

$$
\begin{aligned}
& \underline{\mathcal{C}} \doteq \sum_{k=0}^{T-1}\|c \odot \bar{u}(k)\|_{1}, \\
& \overline{\mathcal{C}} \doteq \sum_{k=0}^{T-1} \sum_{i=1}^{n} c_{i}\left\|\xi_{i}(k)\right\|_{2},
\end{aligned}
$$

with

$$
\xi_{i}(k) \doteq\left[\begin{array}{ll}
\bar{u}_{i}(k) & \boldsymbol{\Theta}_{i}^{\top}(k) \Upsilon(k)
\end{array}\right],
$$

where $\boldsymbol{\Theta}_{i}^{\top}(k)$ denotes the ith row of $\boldsymbol{\Theta}(k)$ and $\Upsilon(k)$ is a full-rank factor such that $\Upsilon(k) \Upsilon^{\top}(k)=\Sigma(k) \doteq \operatorname{var}\{x(k)\}$.

Proof. We first prove the lower bound $\underline{\mathcal{C}} \leq \mathcal{C}$. To this end, we recall that Jensen's inequality (see, e.g., [6]) states that for any random variable $X$ and convex function $f(\cdot)$ it holds that $f(\mathrm{E}\{X\}) \leq \mathrm{E}\{f(X)\}$. Considering in particular $f(\cdot)=|\cdot|$, we have that $|\mathrm{E}\{X\}| \leq \mathrm{E}\{|X|\}$, and thus

$$
\begin{aligned}
\mathcal{C} & =\sum_{k=0}^{T-1} \sum_{i=1}^{n} c_{i} \mathrm{E}\left\{\left|\bar{u}_{i}(k)+\boldsymbol{\Theta}_{i}^{\top}(k) \tilde{\mathbf{g}}(k)\right|\right\} \\
& \geq \sum_{k=0}^{T-1} \sum_{i=1}^{n} c_{i}\left|\mathrm{E}\left\{\bar{u}_{i}(k)+\mathbf{\Theta}_{i}^{\top}(k) \tilde{\mathbf{g}}(k)\right\}\right| \\
& =\sum_{k=0}^{T-1} \sum_{i=1}^{n} c_{i}\left|\bar{u}_{i}(k)\right|=\sum_{k=0}^{T-1}\|c \odot \bar{u}(k)\|_{1}=\underline{\mathcal{C}} .
\end{aligned}
$$

Copyright $\odot$ by SIAM. Unauthorized reproduction of this article is prohibited. 
To prove the upper bound $\mathcal{C} \leq \overline{\mathcal{C}}$, we consider again Jensen's inequality with $f(\cdot)=(\cdot)^{2}$ applied to the random variable $|X|$, which gives $\mathrm{E}\{|X|\} \leq \sqrt{\mathrm{E}\left\{X^{2}\right\}}$, and hence

$$
\begin{aligned}
\mathcal{C} & =\sum_{k=0}^{T-1} \sum_{i=1}^{n} c_{i} \mathrm{E}\left\{\left|\bar{u}_{i}(k)+\mathbf{\Theta}_{i}^{\top}(k) \tilde{\mathbf{g}}(k)\right|\right\} \\
& \leq \sum_{k=0}^{T-1} \sum_{i=1}^{n} c_{i} \sqrt{\mathrm{E}\left\{\left(\bar{u}_{i}(k)+\boldsymbol{\Theta}_{i}^{\top}(k) \tilde{\mathbf{g}}(k)\right)^{2}\right\}}=\sum_{k=0}^{T-1} \sum_{i=1}^{n} c_{i} \sqrt{\bar{u}_{i}^{2}(k)+\boldsymbol{\Theta}_{i}^{\top}(k) D(k) \boldsymbol{\Theta}_{i}(k)} \\
& =\sum_{k=0}^{T-1} \sum_{i=1}^{n} c_{i}\left\|\xi_{i}(k)\right\|_{2}=\overline{\mathcal{C}} .
\end{aligned}
$$

Note that both the upper and lower bounds for the expected transaction cost are convex functions of the decision variables. In particular, $\underline{\mathcal{C}}$ is the sum of absolute values of decision variables, whereas $\overline{\mathcal{C}}$ is the sum of Euclidean norms of linear functions of the decision variables.

4. Convex MAP formulations. The multiperiod allocation problem stated in (2.5), with control (3.2), is written as

$$
\begin{aligned}
& J^{*} \doteq \quad \min _{\bar{u}(k) \in \mathbb{R}^{n}, \boldsymbol{\Theta}(k) \in \mathbb{R}^{n, n k}} \mathcal{R}+\gamma \mathcal{C} \\
& \text { subject to } \quad \mathrm{E}\left\{x^{+}(k)\right\} \in \mathcal{X}(k), k=0, \ldots, T-1 \text {, } \\
& \mathbf{1}^{\top} \bar{u}(k)=0, \quad \mathbf{1}^{\top} \boldsymbol{\Theta}(k)=0, \quad k=0, \ldots, T-1 ; \quad \boldsymbol{\Theta}(0)=0_{n}, \\
& \mathrm{E}\{w(T)\} \geq \psi \cdot w(0) \text {. }
\end{aligned}
$$

Notice that, due to the presence of transaction cost term $\mathcal{C}$, the problem is not yet formulated as a convex program. However, using the convex relaxations developed in the previous section, we can formulate two convex programs that describe upper and lower bounds on $J^{*}$ as formalized in the following proposition.

Proposition 4.1 (convex formulation of MAP). Consider the multiperiod asset allocation problem with affine recourse in (4.1), having optimal value $J^{*}$. We have that

$$
J_{\mathrm{lb}}^{*} \leq J^{*} \leq J_{\mathrm{ub}}^{*},
$$

where $J_{\mathrm{lb}}^{*}$ and $J_{\mathrm{ub}}^{*}$ are, respectively, the optimal values of the following convex optimization problems:

$$
J_{\mathrm{lb}}^{*} \doteq \quad \min _{\bar{u}(k) \in \mathbb{R}^{n}, \boldsymbol{\Theta}(k) \in \mathbb{R}^{n, n k}} \mathcal{R}+\gamma \underline{\mathcal{C}}
$$

subject to $\quad \mathrm{E}\left\{x^{+}(k)\right\} \in \mathcal{X}(k), k=0, \ldots, T-1$,

$$
\begin{gathered}
\mathbf{1}^{\top} \bar{u}(k)=0, \mathbf{1}^{\top} \boldsymbol{\Theta}(k)=0, \quad k=0, \ldots, T-1 ; \quad \boldsymbol{\Theta}(0)=0_{n}, \\
\mathrm{E}\{w(T)\} \geq \psi \cdot w(0),
\end{gathered}
$$

$$
\begin{array}{cc}
J_{\mathrm{ub}}^{*} \doteq & \min _{\bar{u}(k) \in \mathbb{R}^{n}, \boldsymbol{\Theta}(k) \in \mathbb{R}^{n, n k}} \mathcal{R}+\gamma \overline{\mathcal{C}} \\
\text { subject to } & \mathrm{E}\left\{x^{+}(k)\right\} \in \mathcal{X}(k), \quad k=0, \ldots, T-1, \\
& \mathbf{1}^{\top} \bar{u}(k)=0, \mathbf{1}^{\top} \boldsymbol{\Theta}(k)=0, \quad k=0, \ldots, T-1 ; \boldsymbol{\Theta}(0)=0_{n}, \\
& \mathrm{E}\{w(T)\} \geq \psi \cdot w(0),
\end{array}
$$

where $\underline{\mathcal{C}}$ and $\overline{\mathcal{C}}$ are given in (3.10) and (3.11), respectively. 
Remark 2 (alternative formulations). In the current discussion we are considering minimization problems with a mixed objective composed of a risk term $\mathcal{R}$ and of a transaction cost term $\mathcal{C}$ (or bounds on this term). However, many other sensible formulations are possible. For instance, an alternative formulation having the advantage of avoiding the specification of the weight parameter $\gamma$ is to minimize risk while controlling transaction costs via an additional constraint. As an example, one may ask that the total expected cost of transaction $\mathcal{C}$ does not exceed a given fraction $\delta$ of the initial wealth $w(0)$. Since $\mathcal{C} \leq \overline{\mathcal{C}}$, such a condition is guaranteed via the convex constraint $\overline{\mathcal{C}} \leq \delta w(0)$. In this case, the corresponding optimization problem takes the following form:

$$
\begin{array}{cc}
R^{*} \doteq & \min _{\bar{u}(k) \in \mathbb{R}^{n}, \boldsymbol{\Theta}(k) \in \mathbb{R}^{n, n k}} \mathcal{R} \\
\text { subject to } & \mathrm{E}\left\{x^{+}(k)\right\} \in \mathcal{X}(k), \quad k=0, \ldots, T-1, \\
& \mathbf{1}^{\top} \bar{u}(k)=0, \mathbf{1}^{\top} \boldsymbol{\Theta}(k)=0, \quad k=0, \ldots, T-1 ; \quad \boldsymbol{\Theta}(0)=0_{n}, \\
& \mathrm{E}\{w(T)\} \geq \psi \cdot w(0), \\
\overline{\mathcal{C}} \leq \delta \cdot w(0) .
\end{array}
$$

Other variations are possible. For instance, mixed risk/return objectives of the form $\mathcal{R}-\mu \mathrm{E}\{w(T)\} / w(0)$ can be used in the minimization, or return constraints can be imposed at some or all intermediate stages of the optimization horizon. Also, index tracking problems can be easily formulated within this framework. Index tracking problems consider the difference between the portfolio $x(k)$ and an additional asset $b(k)$, which is usually a benchmark or market index, with the objective of minimizing the tracking error volatility $\operatorname{var}\left\{\mathbf{1}^{\top} x(T)-b(T)\right\}$ while guaranteeing that the expected excess return $\mathrm{E}\left\{\mathbf{1}^{\top} x(T)-b(T)\right\}$ is above a given threshold. This setup is cast in our framework simply by considering an augmented portfolio $x_{\text {aug }}(k)=[x(k),-b(k)]$ where the benchmark is shorted $100 \%$ for all $k$.

4.1. Portfolio constraints. In this paper, constraints on portfolio composition are described by a generic set of linear constraints of the form $\mathrm{E}\left\{x^{+}(k)\right\} \in \mathcal{X}(k)$, where $\mathcal{X}(k)$ is a polytope. We next briefly illustrate some typical constraints that can be accommodated within this setting.

Portfolio content constraints. At each period $k$ when the portfolio is rebalanced, constraints on the minimum and maximum expected exposure in an individual security can be imposed, i.e.,

$$
\underline{b}_{i}(k) \leq \mathrm{E}\left\{x_{i}^{+}(k)\right\} \leq \bar{b}_{i}(k), \quad i=1, \ldots, n ; k=0,1, \ldots, T-1,
$$

where $\underline{b}_{i}(k)$ and $\bar{b}_{i}(k)$ are the given lower and upper bounds on portfolio holding in security $a_{i}$ at time $k$ after rebalancing. These limits, as well as the ones described next, are typically assigned by top-level management decisions. For instance, if no shortselling is allowed by management (shortselling is the financial practice of holding a virtually negative amount of an asset), we impose this constraint using the previous bounds, with $\underline{b}_{i}(k)=0$ and $\bar{b}_{i}(k)=\infty$, i.e.,

$$
\mathrm{E}\left\{x_{i}^{+}(k)\right\} \geq 0, \quad i=1, \ldots, n ; k=0,1, \ldots, T-1 .
$$

Portfolio relative diversity constraints. We can limit the fraction of total (posttransaction) wealth held in each asset:

$$
\mathrm{E}\left\{x_{i}^{+}(k)\right\} \leq \nu_{i}(k) \mathbf{1}^{\top} \mathrm{E}\left\{x^{+}(k)\right\}, \quad i=1, \ldots, n ; k=0,1, \ldots, T-1,
$$

where $\nu_{i}(k) \in[0,1]$ is the limit fraction to be invested in security $a_{i}$ at time $k$. 
More generally, we can group the assets in compartments $\left\{c_{1}, \ldots, c_{\ell}\right\}$ where each compartment $c_{j} \subseteq\{1, \ldots, n\}$ contains the indices relative to a group of assets, and impose limits on the fraction of total (posttransaction) wealth held in each compartment:

$$
\begin{aligned}
\nu_{\mathrm{lb}, j}(k) \mathbf{1}^{\top} \mathrm{E}\left\{x^{+}(k)\right\} \leq & \sum_{i \in c_{j}} \mathrm{E}\left\{x_{i}^{+}(k)\right\} \leq \nu_{\mathrm{ub}, j}(k) \mathbf{1}^{\top} \mathrm{E}\left\{x^{+}(k)\right\}, \\
& j=1, \ldots, \ell ; k=0, \ldots, T-1,
\end{aligned}
$$

where $\nu_{\mathrm{lb}, j}(k)$ and $\nu_{\mathrm{ub}, j}(k) \in[0,1]$ are, respectively, the limit lower bound and upper bound fractions to be invested in compartment $c_{j}$ at time $k$.

Remark 3 (constraints on random variables). Notice that, since the portfolio components $x_{i}^{+}(k)$ are random variables that causally depend on past returns, one cannot a priori impose "deterministic" constraints on these values. In this work, uncertain forward constraints are imposed in expectation. The expected value approach has the advantage of preserving linearity of the constraints while imposing them for the average case. While this may seem critical for "hard" constraints, such as the noshortselling conditions, we argue that in practice the optimization model is typically implemented in a sliding-horizon fashion (see section 4.2), and hence only the first "here-and-now" decision and thus the first portfolio $x^{+}(0)$ are actually implemented. Since $x^{+}(0)$ is a deterministic quantity (it does not depend on past returns), the constraints in expectation are met exactly by the implemented portfolio. In this way, imposing constraints in expectation provides a good trade-off between model computability and significance, and guarantees that the actual decisions are feasible in reality, whenever the optimal strategy is implemented in a receding horizon fashion.

4.2. Sliding/shrinking-horizon implementations. In a real-world investment endeavor, multistage decision models are typically implemented using a shrinkinghorizon or a sliding-horizon scheme. Specifically, in a sliding-horizon scheme the length of the decision horizon is kept constant ( $T$ periods). At time $k$ the decision problem is solved over a horizon of $T$ periods forward, and only the first computed adjustment $u(k)$ is actuated. Then, the horizon is moved forward one period, possibly updating the model with new estimates of the conditional gains expectations and covariances, and the process iterates. This approach is useful for continued investments, where a multiperiod model is necessary for obtaining nonmyopic decisions over a certain time scale (say, one month), but the actual investment horizon is much longer (say, years), or it is left unspecified. In a shrinking-horizon scheme, instead, the investor goal is to achieve a target at a precise and fixed final time. In this case, the varying-horizon strategy is implemented by successively reducing the length of the decision horizon as the final time approaches. More precisely, consider, for instance, the following shrinking-horizon version of problem (4.4): At time $k$ (and for $k=0,1, \ldots, T-1)$, we solve problem $\left(\mathcal{P}_{k}\right)$ below

$$
\begin{array}{r}
\left(\mathcal{P}_{k}\right): \quad \min _{\bar{u}(k), \ldots, \bar{u}(T-1) ; \boldsymbol{\Theta}(k+1), \ldots, \boldsymbol{\Theta}(T-1)} \mathcal{R}_{k} \\
\text { subject to } \\
\mathrm{E}\left\{x^{+}(j)\right\} \in \mathcal{X}_{k}(j), \quad j=k, \ldots, T-1, \\
\mathbf{1}^{\top} \bar{u}(j)=0, \quad j=k, \ldots, T-1, \\
\mathbf{1}^{\top} \boldsymbol{\Theta}(j)=0, \quad j=k+1, \ldots, T-1, \\
\mathrm{E}_{k}\{w(T)\} \geq \psi_{k} w(k), \\
\overline{\mathcal{C}}_{k} \leq \delta_{k} \cdot w(k)
\end{array}
$$


and actuate the computed adjustment $u(k)=\bar{u}(k)$. The adjustment needed at $k+1$ is computed at time $k+1$ by re-solving the one-step-ahead version of the above problem and so on. This type of rolling approach is routinely used in model predictive control. A predictive control technique with open-loop model dynamics (as opposed to the closed-loop dynamics considered in the present paper) has also been recently proposed for the purpose of dynamic portfolio selection in [16].

Notice that the expectations and variances appearing in problem (4.5) are denoted by suffix $k$ since they are actually conditioned to the information state at time $k$; that is, they are conditioned upon $g(1), \ldots, g(k)$. Also, the risk objective $\mathcal{R}_{k}$ is the accumulated risk from time $k$ to end of horizon, and $\mathcal{C}_{k}$ represents the upper bound on expected cost of transactions to be executed from time $k$ to time $T-1$.

The advantage of using a sliding- or shrinking-horizon implementation is that at each decision stage we can profit from observations of actual market behavior during the preceding period and use this information, for instance, to feed fresh estimates to the model and to update risk weights and return targets. The way the model is updated at each stage is largely user-dependent, since the decision-maker might decide to arbitrarily modify his/her initial investment profile in the presence of a particular market opportunity/situation.

4.3. Sampling approximations. We now go back to expressions (3.7) and (3.8) for the expected portfolios and wealth variance. Since the parameters $\bar{\Phi}(1, k), \overline{\mathbf{\Phi}}(k)$, $\overline{\boldsymbol{\Psi}}(k)$, and $Q(k)$ appearing in these expressions are not in general expressible in closed form (see section 5 for an important exception), here we propose a standard sampling technique to approximate them. Indeed, we notice that if a stochastic dynamic model is given for the return process $g(k)$, the conditional expectations in (3.7) and the conditional covariance matrix $Q(k)$ can be efficiently approximated by their empirical counterparts. That is, performing $N$ stochastic simulations of the return series $g(t)$, $t=1, \ldots, T$, we may build empirical expectations

$$
\begin{array}{ll}
\hat{\Phi}(1, k)=\frac{1}{N} \sum_{s=1}^{n} \Phi_{s}(1, k), & \hat{\mathbf{\Phi}}(k)=\frac{1}{N} \sum_{s=1}^{n} \boldsymbol{\Phi}_{s}(k), \\
\hat{\mathbf{\Psi}}(k)=\frac{1}{N} \sum_{s=1}^{n} \boldsymbol{\Psi}_{s}(k), & \hat{Q}(k)=\frac{1}{N-1} \sum_{s=1}^{n}\left[\begin{array}{c}
\tilde{\varphi}_{s}(1, k) \\
\tilde{\phi}_{s}(k) \\
\tilde{\psi}_{s}(k)
\end{array}\right]\left[\begin{array}{c}
\tilde{\varphi}_{s}(1, k) \\
\tilde{\phi}_{s}(k) \\
\tilde{\psi}_{s}(k)
\end{array}\right]^{\top},
\end{array}
$$

where the subscript $s$ denotes the value of the subscripted quantity obtained in the sth simulation. By the law of large numbers, the above quantities tend to the actual expectations with probability one as $N$ goes to infinity. In practice, they may represent good approximations of the actual expectations for sufficiently large $N$. Using these approximations, we compute the (approximated) portfolio expectations and wealth variances as

$\hat{x}(k) \doteq \hat{\Phi}(1, k) x(0)+\hat{\boldsymbol{\Phi}}(k) \nu(k)+\hat{\mathbf{\Psi}}(k) \theta(k), \quad$ vâr $\{w(k)\} \doteq\left[\begin{array}{c}x(0) \\ \nu(k) \\ \theta(k)\end{array}\right]^{\top} \hat{Q}(k)\left[\begin{array}{c}x(0) \\ \nu(k) \\ \theta(k)\end{array}\right]$.

Notice that, although simulations are used in order to estimate some parameters of the model, the quantities to be estimated do not depend on the decision variables and can be estimated a priori, that is, before the actual optimization is run. Therefore, this approach does not require the construction of exponentially growing scenario trees. For example, using this proposed sampling approach, the multistage problem (4.2) is 
directly approximated by the following quadratic programming problem (recall that $\nu(k)$ and $\theta(k)$ just contain the variables in $\bar{u}(i)$ and $\boldsymbol{\Theta}(i), i=1, \ldots, k-1$, in vectorized form)

$$
\begin{aligned}
& \hat{J}_{\mathrm{b}}^{*} \doteq \quad \min _{\bar{u}(k) \in \mathbb{R}^{n}, \boldsymbol{\Theta}(k) \in \mathbb{R}^{n, n k}} \hat{\mathcal{R}}+\gamma \underline{\mathcal{C}} \\
& \text { subject to } \quad \hat{x}(k)+\bar{u}(k) \in \mathcal{X}(k), \quad k=0, \ldots, T-1 \text {, } \\
& \mathbf{1}^{\top} \bar{u}(k)=0, \quad \mathbf{1}^{\top} \boldsymbol{\Theta}(k)=0, \quad k=0, \ldots, T-1 ; \quad \boldsymbol{\Theta}(0)=0_{n}, \\
& \hat{w}(T) \geq \psi \cdot w(0), \\
& \text { where } \hat{\mathcal{R}}=\sum_{k=1}^{T} v_{k}\left[\begin{array}{c}
x(0) \\
\nu(k) \\
\theta(k)
\end{array}\right]^{\top} \hat{Q}(k)\left[\begin{array}{c}
x(0) \\
\nu(k) \\
\theta(k)
\end{array}\right] \text {. }
\end{aligned}
$$

We observe that the approximate formulation in (4.7) is to be used when a full stochastic model for return dynamics is available and that this setting allows for consideration of completely general intertemporal statistical dependence in the return process. In the next section, we show that under the widely accepted hypothesis of efficient markets we can actually obtain exact expressions for the portfolio expectations and covariances and hence exact and explicit convex formulations of the multiperiod allocation problem.

5. The efficient market case. In this section, we introduce a standard "efficient market" hypothesis (see $[13,21]$ ) on the return process; that is, we assume that gains over different periods are statistically independent.

Assumption 1 (independent returns). Gain $g_{i}\left(k_{1}\right)$ is statistically independent of $g_{j}\left(k_{2}\right)$ for all $i$ and $j$ and for all $k_{2} \neq k_{1}$.

As we shall see, acceptance of this hypothesis permits analytic recursive expressions for the portfolio expectations and covariances, thus avoiding the need to resort to the sampling approximation of section 4.3. Also, we shall verify that these expressions depend only on the first two conditional moments of the gain vectors $g(k), k=1,2, \ldots$ :

$$
\begin{aligned}
\bar{g}(k) & \doteq \mathrm{E}\{g(k)\}, \quad k=1,2, \ldots, \\
\Sigma(k) & \doteq \operatorname{var}\{g(k)\}=M(k)-\bar{g}(k) \bar{g}^{\top}(k), \quad k=1,2, \ldots,
\end{aligned}
$$

with

$$
M(k) \doteq \mathrm{E}\left\{g(k) g^{\top}(k)\right\}, \quad k=1,2, \ldots,
$$

where the expectations in the previous equations are conditional to past history up to decision time $k=0$.

The following key lemma states explicitly the dynamic equations for expectation and covariances of the controlled portfolio under the independence hypothesis in Assumption 1.

Lemma 5.1 (dynamics of portfolio expectation and covariance). Let Assumption 1 be satisfied, and consider the affinely controlled portfolio equations (3.3) and (3.4). Then, the controlled portfolio expectation $\bar{x}(k)=\mathrm{E}\{x(k)\}$ obeys the recursion

$$
\bar{x}(k+1)=\bar{G}(k+1) \bar{x}^{+}(k), \quad \text { with } \bar{x}^{+}(k)=\bar{x}(k)+\bar{u}(k), \quad k=0, \ldots, T-1,
$$

Copyright $@$ by SIAM. Unauthorized reproduction of this article is prohibited. 
and the portfolio covariance $\Gamma(k) \doteq \mathrm{E}\left\{\tilde{x}(k) \tilde{x}^{\top}(k)\right\}$ follows the recursion

$$
\begin{aligned}
\Gamma(k+1)= & \bar{x}^{+}(k) \bar{x}^{+\top}(k) \odot \Sigma(k+1) \\
& +\left(\Gamma(k)+\boldsymbol{\Theta}(k) D(k) \boldsymbol{\Theta}^{\top}(k)+\Omega(k) \boldsymbol{\Theta}^{\top}(k)+\boldsymbol{\Theta}(k) \Omega^{\top}(k)\right) \\
& \odot M(k+1),
\end{aligned}
$$

initialized with $\Gamma(1)=\bar{x}^{+}(0) \bar{x}^{+\top}(0) \odot \Sigma(1)$, where $D(k) \doteq \operatorname{diag}(\Sigma(1), \ldots, \Sigma(k))$ and $\Omega(k) \doteq \mathrm{E}\left\{\tilde{x}(k) \tilde{\mathbf{g}}^{\top}(k)\right\} \in \mathbb{R}^{n, k n}$ is given for $k=1, \ldots, T-1$ by a parallel recursion

$$
\Omega(k)=\left[\bar{G}(k)(\Omega(k-1)+\boldsymbol{\Theta}(k-1) D(k-1)) \mid \operatorname{diag}\left(\bar{x}^{+}(k-1)\right) \Sigma(k)\right],
$$

initialized with $\Omega(1)=\operatorname{diag}\left(\bar{x}^{+}(0)\right) \Sigma(1)$. For the total wealth variance $\operatorname{var}\{w(k)\}$, it holds that

$$
\begin{aligned}
\operatorname{var}\{w(k+1)\}= & \mathbf{1}^{\top} \Gamma(k+1) \mathbf{1} \\
& =\bar{x}^{+\top}(k) \Sigma(k+1) \bar{x}^{+}(k) \\
& +\operatorname{Tr}\left(\Gamma(k)+\boldsymbol{\Theta}(k) D(k) \boldsymbol{\Theta}^{\top}(k)+2 \boldsymbol{\Theta}(k) \Omega^{\top}(k)\right) M(k+1) .
\end{aligned}
$$

A proof for this lemma is given next. The proof may be safely skipped by the reader not interested in technical details.

Proof of Lemma 5.1. Notice first that, from (3.3) and (3.4) and from the fact that $x^{+}(k)$ and $G(k+1)$ are independent by Assumption 1, we have that the following recursive expressions hold for the expected and centered portfolios:

$$
\begin{aligned}
& \bar{x}(k+1)=\bar{G}(k+1) \bar{x}^{+}(k), \quad \text { with } \bar{x}^{+}(k)=\bar{x}(k)+\bar{u}(k), \\
& \tilde{x}(k+1)=G(k+1)(\tilde{x}(k)+\mathbf{\Theta}(k) \tilde{\mathbf{g}}(k))+\tilde{G}(k+1) \bar{x}^{+}(k),
\end{aligned}
$$

where the first of these recursions is indeed (5.1). We next establish (5.3). Using (5.5) we have that

$$
\begin{aligned}
\Omega(k+1) & =\mathrm{E}\left\{\tilde{x}(k+1) \tilde{\mathbf{g}}^{\top}(k+1)\right\} \\
& =\mathrm{E}\left\{G(k+1)\left[\tilde{x}(k) \tilde{\mathbf{g}}^{\top}(k) \mid \tilde{x}(k) \tilde{g}^{\top}(k+1)\right]\right\} \\
& +\mathrm{E}\left\{G(k+1)\left[\boldsymbol{\Theta}(k) \tilde{\mathbf{g}}(k) \tilde{\mathbf{g}}^{\top}(k) \mid \boldsymbol{\Theta}(k) \tilde{\mathbf{g}}(k) \tilde{g}^{\top}(k+1)\right]\right\} \\
& +\mathrm{E}\left\{\tilde{G}(k+1) \bar{x}^{+}(k) \tilde{\mathbf{g}}^{\top}(k+1)\right\} .
\end{aligned}
$$

We evaluate each of the terms in the previous sum, using the vector identity $\operatorname{diag}(a) b=$ $\operatorname{diag}(b) a$ and observing that Assumption 1 implies that $\tilde{x}(k)$ and $\tilde{\mathbf{g}}(k)$ are independent of $G(k+1)$. We thus have

$$
\begin{aligned}
\mathrm{E}\left\{G(k+1) \tilde{x}(k) \tilde{\mathbf{g}}^{\top}(k)\right\} & =\bar{G}(k+1) \mathrm{E}\left\{\tilde{x}(k) \tilde{\mathbf{g}}^{\top}(k)\right\}=\bar{G}(k+1) \Omega(k) ; \\
\mathrm{E}\left\{G(k+1) \tilde{x}(k) \tilde{g}^{\top}(k+1)\right\} & =\mathrm{E}\left\{\operatorname{diag}(\tilde{x}(k)) \tilde{g}(k+1) \tilde{g}^{\top}(k+1)\right\}=0_{n} ; \\
\mathrm{E}\left\{G(k+1) \boldsymbol{\Theta}(k) \tilde{\mathbf{g}}(k) \tilde{\mathbf{g}}^{\top}(k)\right\} & =\bar{G}(k+1) \boldsymbol{\Theta}(k) D(k) ; \\
\mathrm{E}\left\{G(k+1) \boldsymbol{\Theta}(k) \tilde{\mathbf{g}}(k) \tilde{g}^{\top}(k+1)\right\} & =0_{n} ; \\
\mathrm{E}\left\{\tilde{G}(k+1) \bar{x}^{+}(k) \tilde{\mathbf{g}}^{\top}(k+1)\right\}= & \mathrm{E}\left\{\operatorname{diag}\left(\bar{x}^{+}(k)\right) \tilde{g}(k+1) \tilde{\mathbf{g}}^{\top}(k+1)\right\} \\
& =\left[0_{n} 0_{n} \cdots 0_{n} \mid \operatorname{diag}\left(\bar{x}^{+}(k)\right) \Sigma(k+1)\right],
\end{aligned}
$$

Copyright $@$ by SIAM. Unauthorized reproduction of this article is prohibited. 
which proves (5.3). We now prove (5.2). To this end, we write

$$
\begin{aligned}
\Gamma(k+1) & =\mathrm{E}\left\{\tilde{x}(k+1) \tilde{x}^{\top}(k+1)\right\} \\
& =\mathrm{E}\left\{\tilde{G}(k+1) \bar{x}^{+}(k) \bar{x}^{+\top}(k) \tilde{G}(k+1)\right\} \\
& +\mathrm{E}\left\{G(k+1) h(k) \bar{x}^{+\top}(k) \tilde{G}(k+1)+\tilde{G}(k+1) \bar{x}^{+}(k) h^{\top}(k) G(k+1)\right\} \\
& +\mathrm{E}\left\{G(k+1) h(k) h^{\top}(k) G(k+1)\right\},
\end{aligned}
$$

where for ease of notation we defined

$$
h(k) \doteq \tilde{x}(k)+\mathbf{\Theta}(k) \tilde{\mathbf{g}}(k) .
$$

Note that $h(k)$ is independent of $G(k+1)$. Evaluating each term in the previous sum and observing that for any $Q \in \mathbb{R}^{n, n}$ and vectors $a, b \in \mathbb{R}^{n}$ it holds that $\operatorname{diag}(a) Q \operatorname{diag}(b)=\left(a b^{\top}\right) \odot Q$, we have

$$
\begin{aligned}
\mathrm{E}\left\{\tilde{G}(k+1) \bar{x}^{+}(k) \bar{x}^{+\top}(k) \tilde{G}(k+1)\right\} & =\bar{x}^{+}(k) \bar{x}^{+\top}(k) \odot \Sigma(k+1), \\
\mathrm{E}\left\{G(k+1) h(k) \bar{x}^{+\top}(k) \tilde{G}(k+1)\right\}= & \mathrm{E}\left\{h(k) \bar{x}^{+\top}(k) \odot g(k+1) \tilde{g}^{\top}(k+1)\right\}=0, \\
\mathrm{E}\left\{G(k+1) h(k) h^{\top}(k) G(k+1)\right\} & =\mathrm{E}\left\{h(k) h^{\top}(k) \odot g(k+1) g^{\top}(k+1)\right\} \\
& =\mathrm{E}\left\{h(k) h^{\top}(k)\right\} \odot M(k+1) .
\end{aligned}
$$

Focusing on the term $\mathrm{E}\left\{h(k) h^{\top}(k)\right\}$ in the last expression, we have

$$
\begin{aligned}
\mathrm{E}\left\{h(k) h^{\top}(k)\right\}= & \mathrm{E}\left\{\tilde{x}(k) \tilde{x}^{\top}(k)\right\}+\mathrm{E}\left\{\tilde{x}(k) \tilde{\mathbf{g}}^{\top}(k) \boldsymbol{\Theta}^{\top}(k)+\boldsymbol{\Theta}(k) \tilde{\mathbf{g}}(k) \tilde{x}^{\top}(k)\right\} \\
& +\mathrm{E}\left\{\boldsymbol{\Theta}(k) \tilde{\mathbf{g}}(k) \tilde{\mathbf{g}}^{\top}(k) \boldsymbol{\Theta}^{\top}(k)\right\} \\
= & \Gamma(k)+\mathrm{E}\left\{\tilde{x}(k) \tilde{\mathbf{g}}^{\top}(k)\right\} \boldsymbol{\Theta}^{\top}(k)+\boldsymbol{\Theta}(k) \mathrm{E}\left\{\tilde{\mathbf{g}}(k) \tilde{x}^{\top}(k)\right\} \\
& +\boldsymbol{\Theta}(k) \mathrm{E}\left\{\tilde{\mathbf{g}}(k) \tilde{\mathbf{g}}^{\top}(k)\right\} \boldsymbol{\Theta}^{\top}(k) \\
= & \Gamma(k)+\Omega(k) \boldsymbol{\Theta}^{\top}(k)+\boldsymbol{\Theta}(k) \Omega^{\top}(k)+\boldsymbol{\Theta}(k) D(k) \boldsymbol{\Theta}^{\top}(k) .
\end{aligned}
$$

Substituting back these terms into (5.6) we obtain (5.2) as desired. (5.4) then follows by elementary matrix manipulations, recalling that $\mathbf{1}^{\top}(P \odot Q) \mathbf{1}=\operatorname{Tr} P Q$, if either $P$ or $Q$ is a symmetric matrix.

Remark 4. The expressions of $\bar{x}(k)$ and $\operatorname{var}\{w(k)\}$ resulting from the recursions in Lemma 5.1 can be used in the problem formulations of section 4 to obtain exact and explicit convex formalizations of the MAP problem. These explicit recursions clearly produce a great gain in terms of preprocessing time and absence of approximation errors with respect to the sampling approach discussed in section 4.3. Also, application of these recursions requires only knowledge of the first and second moments of the return vectors, which are indeed the quantities that are usually available from return time series analysis. These exact recursions should therefore be used in place of the sampling approximations whenever the efficient market hypothesis can reasonably be assumed to hold (that is, most of the times in practical asset allocation applications).

6. Numerical tests. Numerical experiments were conducted considering the multiperiod asset allocation problems of section 4 under the efficient market hypothesis. The corresponding optimization programs have been coded into Matlab routines using the Yalmip [20] prototyping interface for convex optimization solvers. Yalmip 
permits us to write an optimization problem much like it is presented on paper. This is particularly important for our specific purposes, since it enables the use of implicit recursive equations in Lemma 5.1 to represent the quantities of interest, without need of explicitly obtaining them. The numerical tests are run under Matlab version 7.4.0 on a quad-Opteron workstation.

6.1. Strategic allocations. As a first example we consider a problem involving strategic investment allocation among two assets and cash over a four-year horizon, with yearly rebalancing. This is a multiperiod problem with $T=4$ periods and $n=3$ asset classes. For the purpose of the numerical test, we considered the estimates for the yearly returns

$$
\bar{g}(1)=\left[\begin{array}{l}
1.0700 \\
1.0350 \\
1.0000
\end{array}\right], \bar{g}(2)=\left[\begin{array}{l}
1.0800 \\
1.0350 \\
1.0000
\end{array}\right], \bar{g}(3)=\left[\begin{array}{l}
1.0900 \\
1.0375 \\
1.0000
\end{array}\right], \bar{g}(4)=\left[\begin{array}{l}
1.0900 \\
1.0375 \\
1.0000
\end{array}\right],
$$

whereas covariance is assumed to increase with periods as follows:

$$
\Sigma(k)=(1+0.1(k-1))\left[\begin{array}{ccc}
0.0100 & -0.0008 & 0 \\
-0.0008 & 0.0016 & 0 \\
0 & 0 & 0
\end{array}\right], \quad k=1,2,3,4 .
$$

In a first test, we set end-of-horizon target return to $20 \%(\psi=1.2)$, objective relative weight $\gamma=1$, risk weights $v_{1}=v_{2}=v_{3}=0$ and $v_{4}=1$ (end-of-horizon risk minimization), and unit transaction costs $c_{1}=0.0020$ for the first two asset classes and $c_{3}=0$ for cash. The initial portfolio is assumed to consist of one unit in cash: $x(0)=\left[\begin{array}{ll}0 & 0\end{array}\right]^{\top}$. No constraints are imposed on the portfolio composition, except for the no-shortselling condition $\mathrm{E}\left\{x^{+}(k)\right\} \geq 0 \forall k$. First, we solve an "open-loop" version of problems (4.2) and (4.3). By open-loop optimization we mean the situation in which all decisions are set beforehand (at the optimization time) and hence no recourse is possible. This situation amounts to zeroing the market reaction matrices, that is, to consider $\boldsymbol{\Theta}(k)=0$ for all $k$. Note that in such a case the upper and lower bounds for the transaction cost are identical, and hence problems (4.2) and (4.3) coincide. The open-loop optimal solution resulted in

$$
\bar{u}_{\mathrm{ol}}(0)=\left[\begin{array}{c}
0.2221 \\
0.7172 \\
-0.9393
\end{array}\right], \quad \overline{\mathrm{ol}}_{\mathrm{ol}}(1)=\left[\begin{array}{c}
0.0260 \\
0.0 \\
-0.0260
\end{array}\right], \quad \bar{u}_{\mathrm{ol}}(2)=\left[\begin{array}{l}
0.0 \\
0.0 \\
0.0
\end{array}\right], \quad \bar{u}_{\mathrm{ol}}(3)=\left[\begin{array}{l}
0.0 \\
0.0 \\
0.0
\end{array}\right],
$$

corresponding to an optimal objective value $J_{\mathrm{ol}}^{*}=0.0092$, with $\mathcal{R}=0.0073$ and $\mathcal{C}=0.0019$.

We next take affine recourse into account, using one-period memory depth in the reaction matrices, and solve problem (4.3), obtaining an optimal solution with nominal adjustments

$$
\begin{aligned}
& \bar{u}_{\mathrm{ub}}(0)=\left[\begin{array}{c}
0.3061 \\
0.6254 \\
-0.9315
\end{array}\right], \bar{u}_{\mathrm{ub}}(1)=\left[\begin{array}{c}
0.0137 \\
0.0430 \\
-0.0567
\end{array}\right], \bar{u}_{\mathrm{ub}}(2)=\left[\begin{array}{c}
-0.0515 \\
-0.0084 \\
0.0599
\end{array}\right], \\
& \bar{u}_{\mathrm{ub}}(3)=\left[\begin{array}{c}
-0.0817 \\
-0.0266 \\
0.1082
\end{array}\right]
\end{aligned}
$$


and market reaction matrices

$$
\begin{aligned}
\Theta_{\mathrm{ub}}(1) & =\left[\begin{array}{ccc}
-0.7228 & -0.9768 & 0 \\
-0.7739 & -1.8746 & 0 \\
1.4968 & 2.8514 & 0
\end{array}\right], \quad \Theta_{\mathrm{ub}}(2)=\left[\begin{array}{ccc}
-1.0138 & -1.5076 & 0 \\
-0.9632 & -2.2291 & 0 \\
1.9771 & 3.7367 & 0
\end{array}\right], \\
\Theta_{\mathrm{ub}}(3) & =\left[\begin{array}{ccc}
-1.2347 & -2.2507 & 0 \\
-0.6873 & -1.6698 & 0 \\
1.9220 & 3.9205 & 0
\end{array}\right],
\end{aligned}
$$

corresponding to an optimal objective value $J_{\mathrm{ub}}^{*}=0.0066$, with $\mathcal{R}=0.0033$ and $\overline{\mathcal{C}}=0.0033$.

Note that in problem (4.3) we overestimate the expected transaction costs, obtaining an upper bound on $J^{*}$. Solving problem (4.2), instead, we underestimate the transaction costs and obtain a lower bound on $J^{*}$. Specifically, an optimal solution to problem (4.2) gives nominal adjustments

$$
\bar{u}_{\mathrm{lb}}(0)=\left[\begin{array}{c}
0.3090 \\
0.6241 \\
-0.9332
\end{array}\right], \quad \bar{u}_{\mathrm{lb}}(1)=\left[\begin{array}{c}
0.0 \\
0.0 \\
0.0
\end{array}\right], \quad \bar{u}_{\mathrm{lb}}(2)=\left[\begin{array}{c}
0.0 \\
0.0 \\
0.0
\end{array}\right], \quad \bar{u}_{\mathrm{lb}}(3)=\left[\begin{array}{c}
-0.12 \\
0.0 \\
0.12
\end{array}\right]
$$

and market reaction matrices

$$
\begin{aligned}
\Theta_{\mathrm{lb}}(1) & =\left[\begin{array}{ccc}
-0.7148 & -0.8195 & 0 \\
-1.2008 & -3.0493 & 0 \\
1.9156 & 3.8689 & 0
\end{array}\right], \quad \Theta_{\mathrm{lb}}(2)=\left[\begin{array}{ccc}
-0.9616 & -1.2327 & 0 \\
-1.7367 & -4.0388 & 0 \\
2.6983 & 5.2715 & 0
\end{array}\right], \\
\Theta_{\mathrm{lb}}(3) & =\left[\begin{array}{ccc}
-1.5432 & -2.2206 & 0 \\
-3.0470 & -6.3732 & 0 \\
4.5902 & 8.5938 & 0
\end{array}\right],
\end{aligned}
$$

corresponding to an optimal objective value $J_{1 \mathrm{~b}}^{*}=0.0050$, with $\mathcal{R}=0.0029$ and $\underline{\mathcal{C}}=0.0021$.

We remark that in this example the solutions obtained from problem (4.3) and from problem (4.2) are qualitatively similar. In problem (4.2) an explicit minimization of a term related to the $\ell_{1}$ norm of $\bar{u}(k)$ is performed, and it is well-known that such minimization tends to produce solutions with many zero elements. This is of course a desirable property which is indeed confirmed in the present numerical experiment. We also notice that problem (4.2) is a convex quadratic programming (QP) problem, whereas (4.3) is a convex second-order-cone program (SOCP, see [18]). Since more efficient optimization solvers exist for QPs than for SOCPs, it might be advisable to first solve the allocation problem with underestimated transaction costs (4.2) and eventually compare its optimal solution to the one of (4.3) to check if the upper-lower bound gap is significant. In the example, the relative gap in optimal objective values is $100 \times\left(J_{\mathrm{ub}}^{*}-J_{\mathrm{lb}}^{*}\right) / J_{\mathrm{ub}}^{*} \simeq 24 \%$. Note also that there is a relevant difference in objective performance between the open-loop (no recourse) and the affine recourse strategy. In particular, comparing the risk levels of the open-loop and recourse strategies (lower bound problem), we see that the open-loop strategy is worse than the recourse strategy by a relative factor of $100 \times(0.0073-0.0029) / 0.0029 \simeq 151 \%$.

6.1.1. Multiperiod efficient frontier. The affine recourse method gives the decision-maker the very valuable possibility of determining an approximation of the multiperiod efficient frontier. This curve is obtained by solving repeatedly the risk minimization problem, with increasing values of the target return. In this example, 
we used the return/covariance data of the previous section and solved repeatedly the affine recourse problem (4.2) with one-period depth and the open-loop (zero depth) version of the same problem (see Remark 1 in section 3 ). The resulting frontiers are displayed in Figure 6.1. This plot shows that, for equal levels of final expected return, the affine recourse strategy provides a relevant reduction in risk level with respect to an open-loop strategy. Note that, in this example, consideration of higher depths in the recourse model provided no further improvement of the frontier.

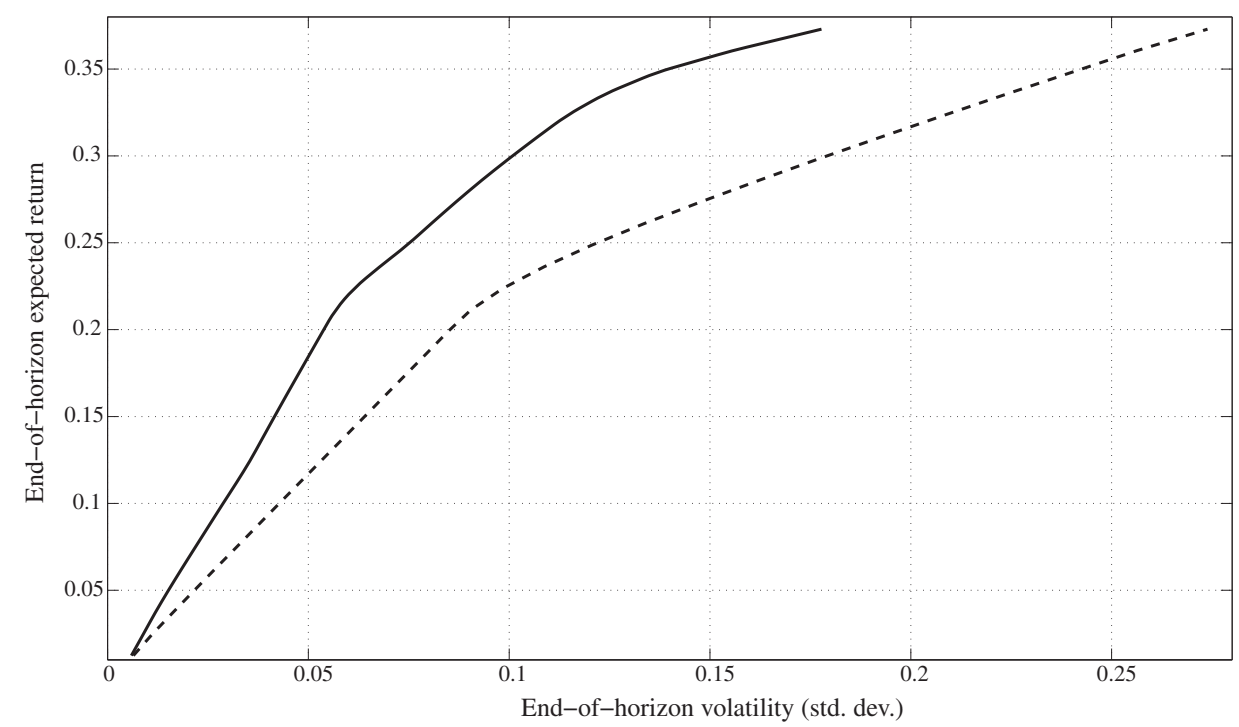

FIG. 6.1. Multiperiod efficient frontiers for the open-loop strategy (dashed line) and the affine recourse strategy with one-period depth (solid line).

6.2. MSCI World index tracking. As a second example, we considered a realistic dynamic allocation problem involving five asset classes, described by four equity indices (1) MSCI USA, (2) MSCI Europe, (3) MSCI Japan, and (4) MSCI Pacific ex Japan and by a monetary index (5) MTS Bot. The objective is to overperform a benchmark index (MSCI World) by dynamically managing a portfolio composed by the five asset classes mentioned above. As discussed in Remark 2, this problem can be cast in our setting by considering an augmented portfolio $x_{\text {aug }}(k)$ composed by the five asset classes and by the benchmark $100 \%$ shorted: $x_{\text {aug }}^{\top}(k)=\left[x^{\top}(k),-b(k)\right]$, where $x^{\top}(k)=\left[x_{1}(k) \cdots x_{5}(k)\right]$ is the portfolio composition and $b(k)$ is the amount virtually invested in the benchmark. We assume that initially $x(0)=\left[\begin{array}{llll}0 & \cdots & 0 & 1\end{array}\right]^{\top}$ and $b(0)=1$. Considering a decision horizon of $T$ periods, the objective is to guarantee that the expected excess return of the portfolio with respect to the benchmark is above a given threshold $\eta, \mathrm{E}\left\{\mathbf{1}^{\top} x_{\text {aug }}(T)\right\} \geq \eta$, while minimizing the tracking error volatility $\operatorname{var}\left\{\mathbf{1}^{\top} x_{\text {aug }}(T)\right\}$.

The purpose of the numerical experiment is to compare the performance obtained by means of the affine recourse multiperiod model against a standard and widely used single-period method. In a single-period model, estimates of the market parameters (expected returns and covariances) are determined at a given time $k$, and an optimal allocation is computed based on a standard Markowitz model on a single period (one month in this example). Then, this portfolio is held unchanged for the whole month, and at the end of the month the process is repeated, etc. This is without a doubt 
the method most frequently used in financial institutions for support of sequential and repeated allocation decisions. Although simple and effective in practice, repeated application of a single-period model may, however, result in myopic investment strategies in the long run. Moreover, it is impossible with single-period methods to provide the investor entering the game at a given time with a long-term view of expected returns and risk associated with his/her investment.

We thus propose to compute allocations using the multistage model on an extended horizon of $T=12$ months. Only the first computed decision $u(0)$ is implemented at each decision time; this portfolio is held for one month, and then the whole process is repeated in a sliding-horizon fashion. In this way, at each decision time (each month) the investor is provided with a portfolio and a perspective plan of investment with a precise target and associated risk over a yearly horizon. We stress the fact that since the strategy is implemented in sliding-horizon, the perspective plan is not actually executed (only the first "here-and-now" decision is implemented), but it is instead recomputed each month for the year forward. In this example, the multistage model assumes that the current estimates of returns and covariance matrices remain constant over the planning horizon. These data are, however, reestimated and updated each time the model is re-solved (i.e., every month). In the single-period model we imposed a target excess return of $0.0417 \%$ monthly, whereas in the multiperiod model we directly impose a long-term target excess return of $0.5 \%$ at the end of the year. Bounds are imposed on the portfolio composition (in both the single-period and the multiperiod models) in order to consider portfolios that do not divert too much from the composition of the index to be tracked. The bounds are $\underline{b}=\left[\begin{array}{lllll}0.4 & 0.2 & 0 & 0 & 0\end{array}\right]^{\top}$ and $\bar{b}=\left[\begin{array}{lllll}0.6 & 0.4 & 0.25 & 0.14 & 0.15\end{array}\right]^{\top}$. Historical asset data have been kindly provided by ERSEL Asset Management SGR SpA, Torino (www.ersel.it). ERSEL also provided a proprietary model for estimation of expected returns and covariances based on a 150-week-rolling data batch. Back-testing is executed on a monthly basis from January 1993 to March 2007 (170 months). Simulation results in Figure 6.2 show

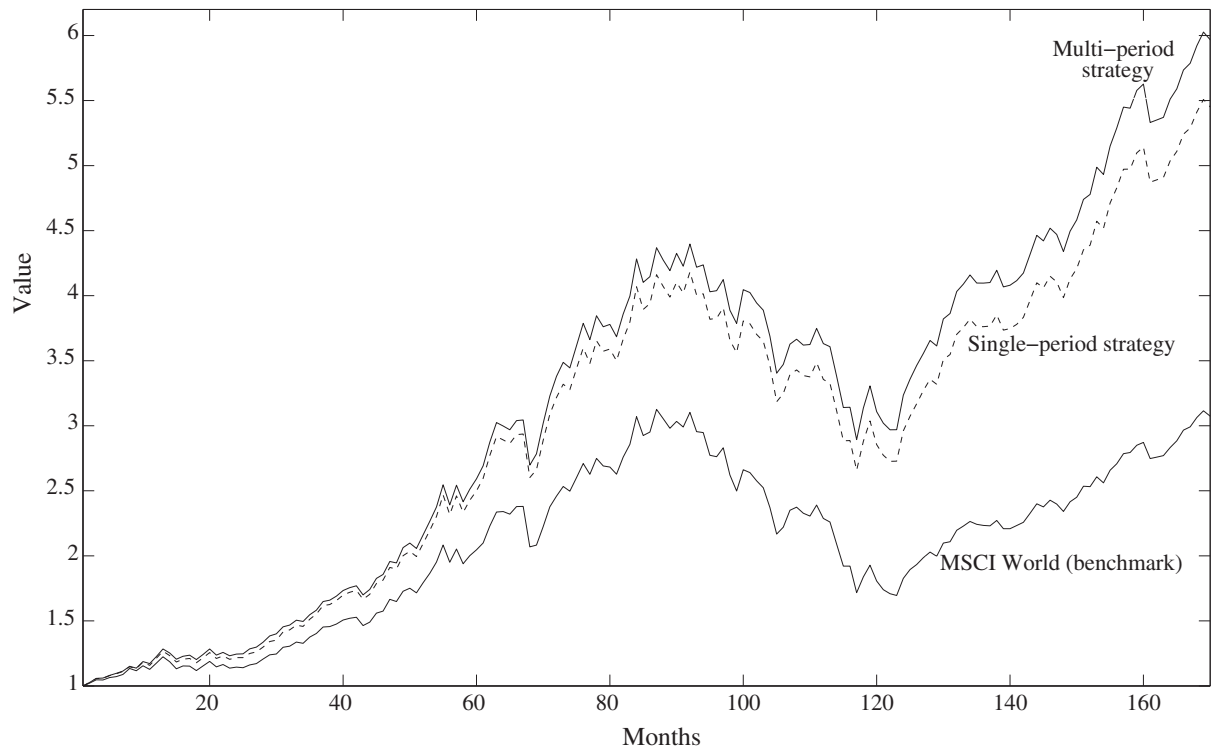

FIG. 6.2. Time value of one dollar invested in benchmark index and in the single-period and multiperiod strategies. 
that the use of a multiperiod decision model actually provided a long-term advantage over the single-period one.

7. Conclusions. In this paper, we presented a mean-variance computational model for dynamic asset allocation over multiple discrete-time periods. The main features of the model are (a) the flexibility of dealing with interperiod and end-ofhorizon constraints on portfolio expectations and covariances, (b) the inclusion of proportional transaction costs, (c) the generality of stochastic return models that can be used with the method (for instance, nonstationary and possibly time-correlated return processes), and (d) the efficiency in numerical solution, which is derived by the finite-dimensional convex representation of the problem. The method completely avoids stochastic approximations via scenario trees. Also, in the efficient market case, the proposed approach provides explicit analytic recursions for the statistics of the controlled portfolio. Moreover, the affine parameterization of the control law provides the user with a compact representation which brings a sound interpretation in terms of nominal corrective actions and sensitivity (gains) with respect to market deviations from expected behavior.

The mentioned strong points are achieved at the expense of a degree of suboptimality of the method, since an affine restriction on the class of reaction functions is imposed a priori. It should, however, be remarked that a method for efficient and exact computation of the actual optimal solution of the considered problem is to date unavailable. Also, approximate techniques such as stochastic programming currently appear to be able to deal only with problems with few assets and very few periods. The proposed technique could instead be employed with success in practical medium-sized problems involving a number of assets and periods at least of the order of tenths.

\section{REFERENCES}

[1] B. R. BARMISH, On trading of equities: a robust control paradigm, in Proceedings of the 17th IFAC World Congress, Seoul, Korea, 2008.

[2] A. Ben-Tal, A. Goryashko, E. Guslitzer, and A. Nemirovski, Adjustable robust solutions of uncertain linear programs, Math. Program. Ser. A, 99 (2004), pp. 351-376.

[3] J. R. Birge, Multistage Stochastic Planning Models Using Piecewise Linear Response Functions, Technical report UMR4802, University of Michigan, Ann Arbor, MI, 1989.

[4] J. R. BIRGE, Stochastic programming computation and applications, INFORMS J. Comput., 9 (1997), pp. 111-133.

[5] J. R. Birge and F. Louveaux, Introduction to Stochastic Programming, Springer Ser. Oper. Res., Springer, New York, 1997.

[6] S. Boyd and L. Vandenberghe, Convex Optimization, Cambridge University Press, Cambridge, 2004.

[7] M. W. BrandT, Estimating portfolio and consumption choice: a conditional Euler equations approach, J. Finance, 54 (1999), pp. 1609-1645.

[8] M. J. Brennan, E. S. Schwartz, and R. Lagnado, Strategic asset allocation, J. Econom. Dynam. Control, 21 (1997), pp. 1377-1403.

[9] G. C. CAlafiore, Multi-period portfolio optimization with linear control policies, Automatica J. IFAC, 44 (2008), pp. 2463-2473.

[10] O. L. V. Costa AND R. B. NABholz, Multiperiod mean-variance optimization with intertemporal restrictions, J. Optim. Theory Appl., 134 (2007), pp. 257-274.

[11] G. B. Dantzig And G. Infanger, Multi-stage stochastic linear programs for portfolio optimization, Ann. Oper. Res., 45 (1993), pp. 59-76.

[12] Y. ERmoliev and R. J.-B. Wets, EDS., Numerical Techniques for Stochastic Optimization, Springer Ser. Comput. Math. 10, Springer, New York, 1988.

[13] E. F. FAMA, Efficient capital markets: A review of theory and empirical work, J. Finance, 25 (1970), pp. 383-417. 
[14] N. Gulpinar, B. Rustem, And R. Settergren, Multistage stochastic programming in computational finance, in Computational Methods in Decision-Making, Economics and Finance, Appl. Optim., Kluwer Academic Publishers, Dordrecht, 2002, pp. 33-47.

[15] F. Herzog, G. Dondi, And H. Geering, Stochastic model predictive control and portfolio optimization, Int. J. Theoret. Appl. Finance, 10 (2007), pp. 203-233.

[16] F. Herzog, S. Keel, G. Dondi, L. M. Schumann, and H. P. Geering, Model predictive control for portfolio selection, in Proceedings of the American Control Conference, Minneapolis, IFAC, 2006.

[17] N. HiBIKI, Multi-period stochastic optimization models for dynamic asset allocation, J. Banking Finance, 30 (2006), pp. 365-390.

[18] M. S. Lobo, L. Vandenberghe, S. Boyd, and H. Lebret, Applications of second-order cone programming, Linear Algebra Appl., 284 (1998), pp. 193-228.

[19] J. Löfberg, Minimax Approaches to Robust Model Predictive Control, Ph.D. thesis, Linköping University, Linköping, Sweden, 2003.

[20] J. LöfBerg, Yalmip: A toolbox for modeling and optimization in MATLAB, in Proceedings of the Computer Aided Control System Design (CACSD) Conference, Taipei, Taiwan, IEEE, 2004.

[21] B. G. Malkiel, Efficient market hypothesis, in The New Palgrave: A Dictionary of Economics, Vol. 2, M. Milgate, J. Eatwell, and P. Newman, eds., Macmillan \& Stockton, London and New York, 1987, pp. 120-123.

[22] R. Mansini, W. Ogryczak, and M. G. Speranza, Conditional value at risk and related linear programming models for portfolio optimization, Ann. Oper. Res., 152 (2007), pp. 227-256.

[23] H. M. Markowitz, Portfolio selection, J. Finance, 7 (1952) pp. 77-91.

[24] H. M. Markowitz, Portfolio Selection, Blackwell, Malden, MA, 1991.

[25] R. C. Merton, Optimum consumption and portfolio rules in continuous time model, J. Economic Theory, 3 (1971), pp. 373-413.

[26] R. C. Merton, An intertemporal asset pricing model, Econometrica, 41 (1973), pp. 867-887.

[27] G. Ch. Pflug, Scenario tree generation for multiperiod financial optimization by optimal discretization, Math. Program., 89 (2001), pp. 251-271.

[28] M. PINAR, Robust scenario optimization based on downside-risk measure for multi-period portfolio selection, OR Spectrum, 29 (2007), pp. 295-309.

[29] J. Primbs, Portfolio optimization applications of stochastic receding horizon control, in Proceedings of the IEEE American Control Conference, New York, 2007.

[30] B. Rustem and N. Gulpinar, Worst-case robust decisions for multi-period mean-variance portfolio optimization, European J. Oper. Res., 183 (2007), pp. 981-1000.

[31] A. Ruszczyński And A. Shapiro, eds., Stochastic Programming, Handbooks Opers. Res. Management Sci., Elsevier, Amsterdam, 2003.

[32] A. Shapiro, On complexity of multistage stochastic programs, Oper. Res. Lett., 34 (2006), pp. 1-8.

[33] A. Shapiro, D. Dentcheva, and A. Ruszczynski, Lectures on Stochastic Programming: Modeling and Theory, SIAM, Philadelphia, 2009.

[34] M. C. SteinBach, Markowitz revisited: mean-variance models in financial portfolio analysis, SIAM Rev., 43 (2001), pp. 31-85.

[35] W. T. Ziemba And J. M. Mulvey, eds., Worldwide Asset and Liability Modeling, Cambridge Univ. Press, Cambridge, 1998.

Copyright (c) by SIAM. Unauthorized reproduction of this article is prohibited. 